\title{
Velocity Measurements of Gap Flows at a Computer Hard Disk Model
}

Shirai, Katsuaki; Büttner Lars; Czarske, Jürgen

Technische Universität Dresden

Laboratory of Measurement and Testing Techniques

Helmholtzstraße 18, D-01069 Dresden, Germany

Tel.: +49-351-463-37657, Fax: +49-351-463-37716

E-Mail: juergen.czarske[at]tu-dresden.de

Obi, Shinnosuke

Keio University

Department of Mechanical Engineering

3-14-1 Hiyoshi, Kouhoku-ku, Yokohama 223-8522, Japan

Tel.: +81-45-566-1499, Fax.: +81-45-566-1495

E-Mail: obsn[at]mech.keio.ac.jp

\section{Background and Research Purpose}

Hard disk drives (HDDs) are among the most widely spread data storage devices. The development of HDDs goes toward higher data densities and increased rotation speeds with a continuously growing demand for larger storage capacity and faster data access speed. On the other hand, the next generation of HDDs is expected to be smaller in size and to consume less energy. The distance between the read-and-write head and the disk surface reaches into the range of $10 \mathrm{~nm}$ for recently developed HDDs. The disk rotation entrains the surrounding air and lets threedimensional complex flow structures develop in a HDD. The complex flow induces disk vibrations, which may lead irreparable damage to the disks. Many investigations have been reported on the disk vibrations or/and on the flow structures in a HDD, but the detailed mechanism of the flow-disk interaction remains still yet to be understood.

The air flow in a HDD can be roughly divided into three different regions: inner, outer and shroud regions [1] as depicted in Fig. 1. Region I $\left(0 \leq r_{d} / R_{2} \leq 0.4\right)$ is the "inner region" where flow exhibits the solid body rotation $\left(r_{d}\right.$ : the radial coordinate originating from the spindle exterior, $R_{2}$ : the disk radius). Region II $\left(0.4 \leq r_{d} / R_{2} \leq 0.75\right)$ is the "outer region" where distinct vortical structure is observed. Region III $\left(0.75 \leq r_{d} / R_{2}\right)$ is the "shroud region" where significant turbulent production occurs due to the strong shear at the shroud wall.

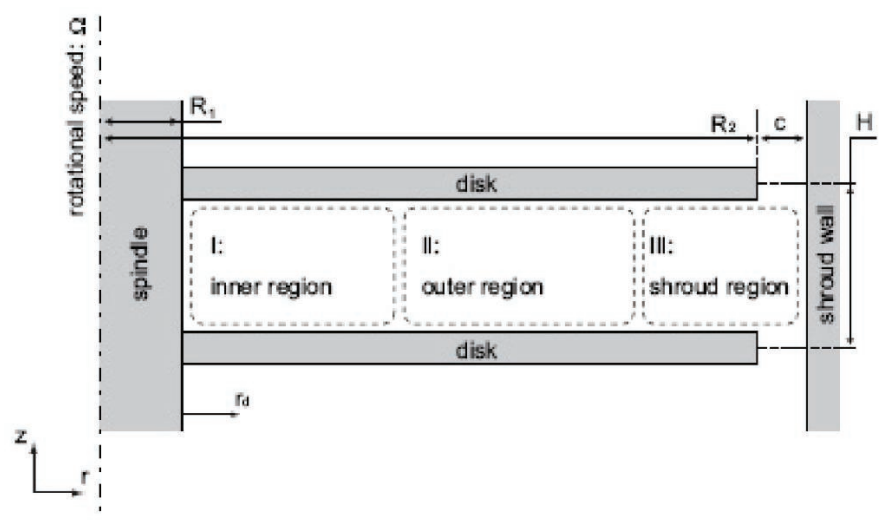

Fig. 1: Sketch of the flow regions in a co-rotating disk system.

Most of the investigations in the past studied the flow in the large area between the disks (inner and outer regions in Fig. 1), but almost no one has ever focused on the flow in the region near the shroud (shroud region in Fig. 1). The disk vibration has been known to decrease either by narrowing the tip clearance between the disk and the shroud [2] or by using a shroud wall with a ribbed shape [3]. The reduction mechanism of the disk vibration needs to be clarified and the flow inside the tip clearance region is of greatest interest in this respect. In spite of the interest, experimental investigation remains challenging. Especially, the flow inside the gap between the disk and shroud should exhibit highly three-dimensional complex structures due to the mean shear forces induced along both the axial and the radial directions accompanying the disk rotation. A sophisticated measurement technique is required so as not to disturb the flow while simultaneously achieving high spatial resolution at least some tens of micrometers required in the region. None of the conventional measurement techniques afford these requirements. Probe based methods such as hot-wire anemometry would disturb the flow and the spatial resolution would be critical. Particle image velocitmetry is also difficult to be applied since the area of interest is very small with the high shear rate induced in two directions.

In the present study, we focus on the region of tip clearance which is a part of the shroud region. Our approach to the flow is using a novel laser Doppler velocity profile sensor [4, 5] in order to overcome the aforementioned difficulties. In contrast to a conventional laser Doppler velocimetry (LDV), the profile sensor employs a pair of fanlike fringe systems superposed in a single measurement volume consisting of diverging and converging fringe 
systems along the optical axis. This allows the determination of not only the lateral velocities but also the axial positions of single tracer particles inside the measurement volume, and hence the velocity profile inside the measurement volume is reproduced with a high spatial resolution. The sensor has a sub-micrometer spatial resolution [6] and a small measurement uncertainty of velocities [7]. It has been successfully applied to a number of flow investigations ranging from microfluidics to turbulent flows $[6,7,8,9]$.

We describe the main findings on the flow in the narrow gap region between the disk and the shroud of a scaled experimental model of a HDD. The details of the investigation is reported in another paper [10]. The measurements were carried out both with an LDV and with a velocity profile sensor. The main purpose was to gain the fundamental insight on the complex turbulence behaviors in the tip clearance region. For this reason, an axissymmetric model was used without any disturbances and assymmetry such as read-and-write arm and the shroud opening existing in a real HDD. The spatial averaging effect was examined at first using the different spatial resolutions of the sensors on the resulting turbulence statistics. The flow behaviers were investigated at four different configurations with the combination of two different tip clearances and two different geometrical configurations of the shroud walls with and without ribs.

\section{Experimental Apparatus and Instrumentation}

\section{HDD Model}

Flow measurements were performed with a 3.4 times scaled-up, simplified axissymmetric experimental model for simulating a HDD as shown in Fig. 2. For the details, another paper can be referred [10]. The disks and enclosure were made of polymethyl-methacrylate (PMMA) except for the optical windows made of glass. The disks were connected to a DC motor through the enclosure bottom. The enclosure was filled with tap water as working fluid. The flow behavior was investigated at four different test cases with two different tip clearances of $c=5 \mathrm{~mm}$ and 2 $\mathrm{mm}$ and with two different shroud peripheral shapes termed "flat" (F) and "ribbed" (R), respectively. These tip clearances corresponded to $1.5 \mathrm{~mm}$ and $0.6 \mathrm{~mm}$, and the latter is equivalent to the clearance of a commercially available HDD. The flat shroud had a smooth inner surface and the ribbed shroud had five ribs as depicted in Fig. 3. These four test cases are denoted by the case names: F2, F5, R2 and R5 (see Table 1). The rotation speed was adjusted so that the resulting disk Reynolds number remained constant for all of the experiments. The disk Reynolds number $\operatorname{Re}_{D}=R_{2}{ }^{2} \Omega / v$ based on the angular velocity $\Omega$, the disk radius $R_{2}$ and the kinematic viscosity of water $v$, was set to $1.4 \times 10^{5}$ corresponding to $10,000 \mathrm{rpm}$ in a typical 3.5-in HDD.

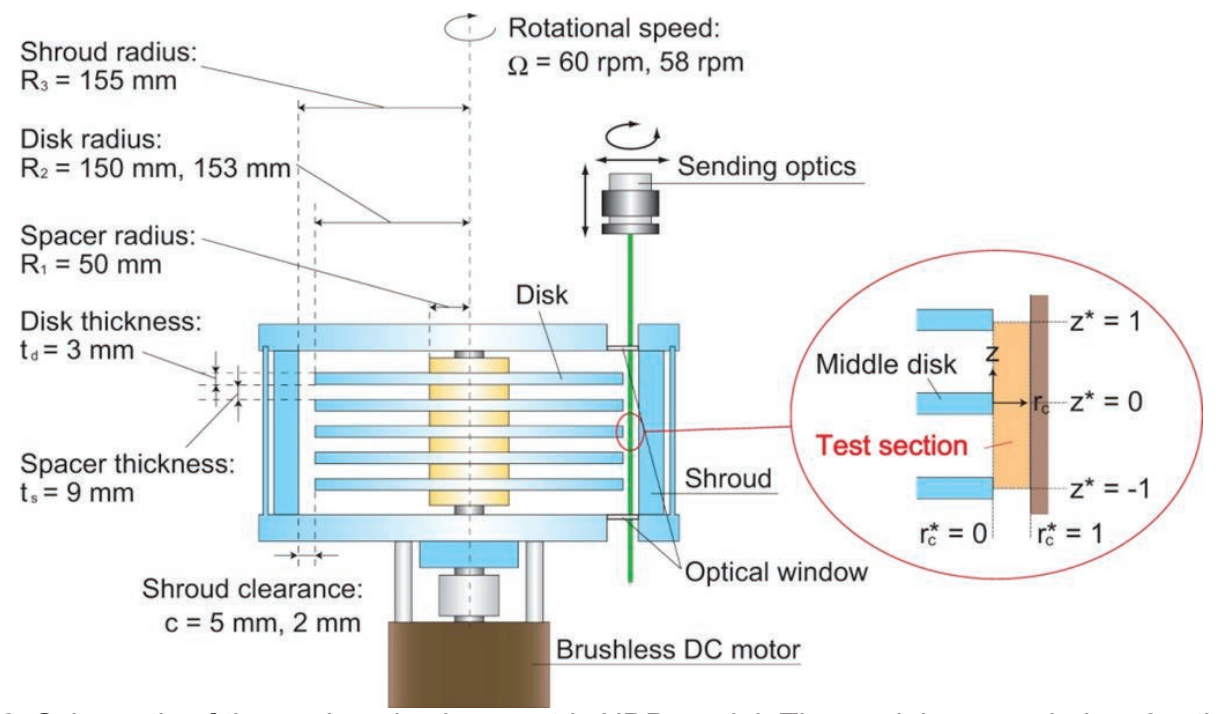

Fig. 2: Schematic of the enclosed axisymmetric HDD model. The model was scaled up 3.4 times of a 3.5-in commercial HDD and it was filledwith water as working fluid. The flow was driven by the motor attached at the bottom. The disks and the shrouds were exchangeable in order to realize the different experimental conditions (see Tab. 1).

The test section was located in the region near the tip clearance shown in the inset of Fig. 2 . The measurements were undertaken in the $r_{c}-z$ plane. The positions are normalized by the tip clearance $c$ and the center-to-center distance $H$ between two neighboring disks, respectively. The normalized radial position $r_{c}$ is set such that $r_{c}=0$ refers to the outermost position of the disk (disk tip) and $r_{c}=1$ to the position of the inner shroud wall for the flat shroud case or the innermost position of the ribs for the ribbed shroud case. The normalized axial position $z$ is set so that $z^{*}=0$ refers to the central plane of the middle disk and $z^{*}=1$ refers to the center of the overlying disk. The circumferential $V_{\theta}^{*}\left(=V_{\theta} / R_{2} \Omega\right)$ and radial $V_{r}^{*}\left(=V_{r} / R_{2} \Omega\right)$ velocities are normalized with the circumferential velocity of the disk tip, respectively. We refer to different regions of flow in the test section as "tip clearance", "inter disk region" and "inter rib region" shown in Fig. 3. All measurements presented in this paper were obtained in the area around the third disk located at the middle in order to avoid singularity originating from the top and bottom of the casing. Modern commercial HDDs have various configurations including the number of disks. The flow in the tip clearance of the third disk, on which we focus, is expected to be similar to that of other disks. 

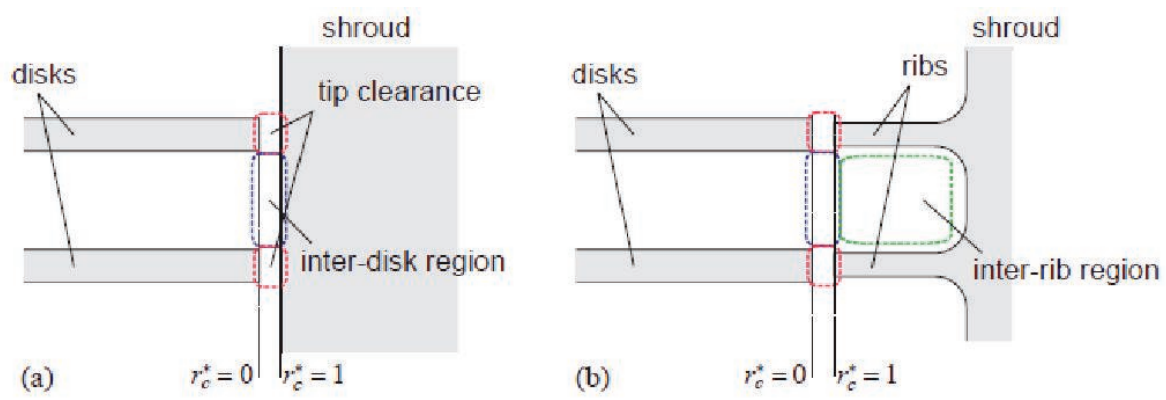

Fig. 3: Two different shroud shape configurations and the definition of the regions investigated:

(a) flat-shaped shroud, (b) ribbed-shaped shroud.

\section{Sensors}

A commercial fiber-optic one-component LDV system was used as a reference technique. The fiber-optic measurement head had a working distance of $250 \mathrm{~mm}$. The estimated dimensions of the measurement volume were $84 \mu \mathrm{m}$ and $1.6 \mathrm{~mm}$ in diameter and length in water, which determines the spatial resolution of the LDV measurement. A hardware based signal processor was equipped for processing the burst signals in the single-burst mode. The systematic measurement uncertainty was estimated around $0.6 \%$ for the present LDV system, which is ineviatble even for a perfectly aligned system due to the Gaussian nature of the laser beams.

The velocity profile sensor used in the present study was based on a frequency-division-multiplexing (FDM) technique, with which the two fringe systems are distinguished by two different carrier frequencies. The details of the FDM sensor system were described in [11]. The fiber-optic measurement head had a working distance of about $560 \mathrm{~mm}$. The dimensions of the measurement volume were approximately $200 \mu \mathrm{m}$ and $2000 \mu \mathrm{m}$ in diameter and length in water. The circumferential component of the flow velocity was measured with a high spatial resolution in the axial direction. The sensor was calibrated in-situ using a pinhole attached to a specially-made calibration disk which was exchangeable with the third disk. From the calibration data, the spatial resolution of the sensor was estimated to be approximately $22 \mu \mathrm{m}$ along the $z$ axis in water and the resulting theoretical inherent uncertainty of the velocity measurements was $0.04 \%$.

\section{Measurement and Results}

\section{Measurement Conditions}

We have tested four different configurations of the disks and shroud shapes comprising combinations of flat or ribbed shroud shapes and gap widths of $5 \mathrm{~mm}$ or $2 \mathrm{~mm}$. Hereafter, these different cases are denoted by the names of the cases: F5, F2, R5 and R2 as listed in Table 1.

Table 1: Measurement conditions. (The tip clearance is the dimensions at the model.)

\begin{tabular}{c|c|c} 
case & tip clearance $[\mathrm{mm}]$ & shroud wall type \\
\hline F5 & 5 & flat \\
F2 & 2 & ribbed \\
R5 & 5 & flat \\
R2 & 2 & ribbed
\end{tabular}

For the velocity measurements with the LDV, forward-scattered signals were detected in the backward direction using a retroreflector in order to enhance the signal quality. Naturally occurring dust in the water functioned as tracer particles without adding specific tracer particles. At least 5000 samples were acquired at each location. The velocity statistics were calculated using bias correction based on transit-time weighting.

For the measurements with the velocity profile sensor, backward scattering was mainly used and forward-scattering detection was used only for regions inaccessible by backscatter detection. Silver-coated hollow glass spheres with a mean diameter of $10 \mu \mathrm{m}$ were used as tracer particles. The measurement volume was traversed with an increment of $1.6 \mathrm{~mm}$ along the axial direction with an overlap of $0.4 \mathrm{~mm}$ for every neighboring measurement location. From preliminary LDV measurements, the axial distributions of the velocity variance was found to be almost symmetric to the disk middle plane $\left(z^{*}=0\right)$ in the range of $-1 \leq z^{*} \leq 1$. In addition, the variance was found to be almost symmetric with respect to the plane of $z^{*}=0.5$ in the range of $0 \leq z^{*} \leq 1$. Hence, the velocity distributions in the test section, $0 \leq r_{c} \leq 1$ and $-0.2 \leq z^{*} \leq 0.7$, were measured with the velocity profile sensor. Roughly 35000 samples were collected at every measurement location.

The velocity statistics (mean velocities, velocity variances) were calculated using slot statistics from the raw position-velocity data of the velocity profile sensor [23]. Hence, the resulting practical spatial resolution of the statistics was determined by the slot width applied to each calculation. Throughout the present investigation, the resulting statistics were calculated from more than 2000 samples per slot without any correction scheme for velocity bias. The minimum slot width set for the statistical calculations was determined to be $100 \mu \mathrm{m}$ for having sufficient samples in each slot, while the slot width of $100 \mu \mathrm{m}$ was indeed larger than the theoretical spatial resolution of the 
sensor itself $\left(\sigma_{z}=22 \mu \mathrm{m}\right)$. The statistics are calculated using different slot widths in order to examine if the slot width is sufficient for resolving the expected flow structures in the region.

\section{Spatial Resolution and Turbulence Statistics}

Fig. 4 shows statistics calculated with different slot widths $(100,300,1600 \mu \mathrm{m})$ for an identical set of the raw data. The results of the LDV measurement are shown together in the same plots. The statistics calculated with a slot width of $1600 \mu \mathrm{m}$ correspond to the spatial resolution of the LDV in the axial direction. Fig. 4 (a) clearly indicates that the two velocity peaks captured with the velocity profile sensor using the small slot widths were not captured by the LDV. The large slot width of $1600 \mu \mathrm{m}$ corresponding to the axial resolution of the LDV also failed to capture the two peaks. A slot width of $300 \mu \mathrm{m}$ or smaller is required to see the two peaks. For the proper determination of the slot width, one should look at the velocity variance. This is because the velocity variance varies depending on the slot width size while the contribution of the sensor spatial resolution remains constant regardless of the slot width. Fig. 4 (b) shows the velocity variance calculated for different slot widths. Clearly the LDV failed to capture the behavior of the velocity variance because it already failed to trace the behavior of the mean velocity profile (see Fig. 4 (a)). The variance value calculated for the results of the velocity profile sensor shows a strong dependence on the slot width. The variance calculated for a fixed location shows larger values for larger slot widths due to the spatial averaging effect. The variance for the slot width of $1600 \mu \mathrm{m}$ results in excessively large variance values at the locations where the velocity gradient is steep. Those calculated with a moderately sized slot width of $300 \mu \mathrm{m}$ still shows rather high values compared to those calculated with the $100 \mu \mathrm{m}$ slot width. By decreasing the slot width, the variance profile for a slot width of $100 \mu \mathrm{m}$ shows little difference compared to that for a $300 \mu \mathrm{m}$ slot width. However, the profile calculated with the $100 \mu \mathrm{m}$ slot width starts to show statistical scattering due to the relatively small number of samples contained in each slot. Therefore, the slot width of $100 \mu \mathrm{m}$ should be an appropriate choice for calculating the velocity statistics without being seriously influenced by the slot witdht through spatial averaging effect in the present study. Indeed, the spatial averaging occurs more significantly on the higher order velocity statistics through the subtraction of the spatially insufficiently resolved mean velocities from the raw velocities. The details of the discussion on the spatial averaging is found in [10].
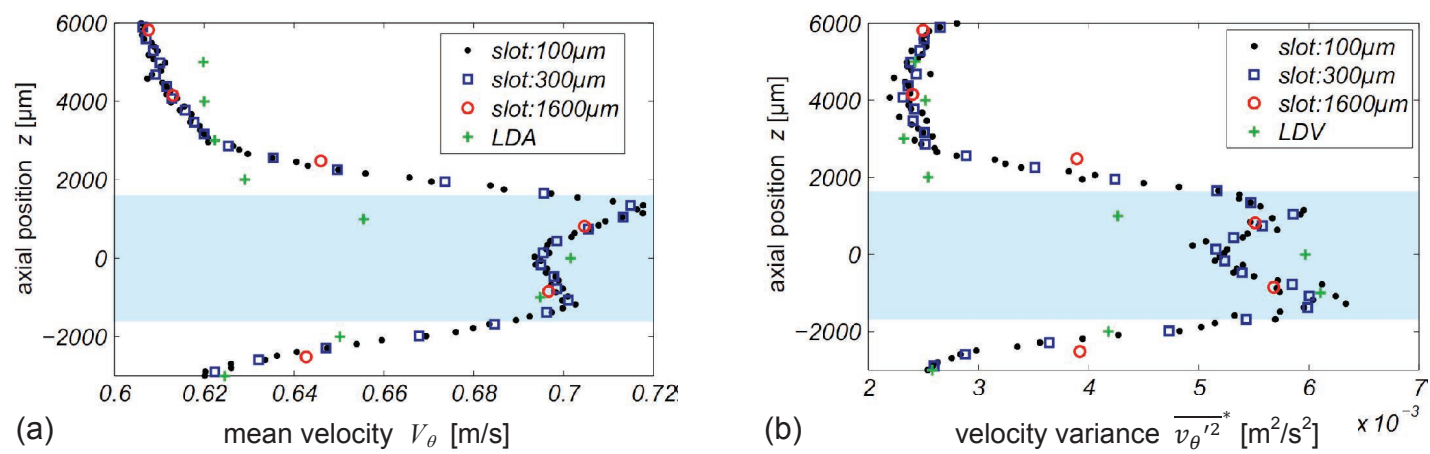

Fig. 4: Effect of the slot width on the resulting statistics: (a) mean velocity; (b) velocity variance. I he statistics are shown on a real scale without nondimensionalization. The LDV results are also plotted as a reference. The disk position is indicated by the shaded area.

\section{Mean Velocity}

Two-dimensional distributions of the circumferential mean velocity $V_{\theta}$ are reconstructed using the statistics with 100 $\mu \mathrm{m}$ slots in the axial direction with the radial positions fixed to the measurement locations. The normalized velocity distribution is plot with color codes in Fig. 5. The four distributions at the different conditions clearly exhibit the existence of velocity gradients in two directions, namely, the radial and axial directions. The distributions show some difference for the different gap widths and shroud shapes. The two peaks are clearly observed in the cases of the $5 \mathrm{~mm}$ gap width in Fig. $5(\mathrm{a}, \mathrm{c})$ while the peaks for the cases of the $2 \mathrm{~mm}$ gap width are less obvious in Fig. 5 $(b, d)$. These peaks correspond to the disk edges, shearing the fluid strongly in the tip clearance region. In the cases of the flat shrouds, the mean flow exhibits prominently different structures for the case of the 5 mm gap width in Fig. 5 (a) and for that of the $2 \mathrm{~mm}$ gap one in Fig. 5 (b). In the case of the $2 \mathrm{~mm}$ gap width, a strong interaction is observed in the tip clearance region, while such an interaction is not observed in the case of the $5 \mathrm{~mm}$ gap width. The interaction is caused by two boundary layers develop over different surfaces schematically depicted in Fig. 6 . In the case of the $2 \mathrm{~mm}$ gap width, the boundary layer develop over the disk top and bottom surfaces reaches into the tip clearance region, and the one develop around the shroud wall intrudes into the inter-disk region (Fig. 6 (b)). Compared to the cases of the flat shrouds, no large difference is observed in the inter-disk region for the cases of the ribbed shrouds.

\section{Velocity Variance}

The normalized distributions of the circumferential velocity variance ${\overline{v_{\theta}}}^{*}$ are plot with color codes in Fig. 7. The distributions were reconstructed in a similar way as for the mean velocity ones using the slotted statistics with 100 $\mu \mathrm{m}$ slot width. The peaks of the velocity variances mostly correspond to the large gradients observed in the mean velocity in Fig. 5. The distributions of the velocity variance show different features for the different gap widths and shroud shapes. The interactions of the two boundary layers observed in the mean flow also appear in the velocity variances for the cases of the $2 \mathrm{~mm}$ gap widths in Fig. 7 (b, d). In contrast, they exhibit non-active region between 
the disk and the shroud in the tip clearance region for the cases of $5 \mathrm{~mm}$ gap widths in Fig. 7 (a, c). In terms of the different shroud shapes, the ribbed shrouds seem to induce strong shear in the axial direction in the tip clearance region, while the variance remains small in the inter-disk region. This can be explained by the large area grooved in the inter-rib region, where the fluid is accommodated. The velocity variances in the cases of the flat shrouds exhibit prominent feature of the turbulence in the tip clearance region for the case of the $2 \mathrm{~mm}$ gap width in Fig. 7 (b) compared to that of the $5 \mathrm{~mm}$ gap in Fig. 7 (a). The boundary layer developed at the top and bottom sides of the disk surfaces seem to penetrate into the tip clearance region, and hence, the peaks of the velocity variance exhibits two elongated patterns both in the axial and radial directions near the shroud wall for the case of the $2 \mathrm{~mm}$ gap width. The velocity variances in the cases of the ribbed shrouds show uniformly small magnitudes outside the tip clearance region in Fig. 7 (c, d) compared to those in the flat shrouds in Fig. 7 (a, b). Hence, the use of the ribbed shrouds does not reduce the turbulence production in the whole flow field.
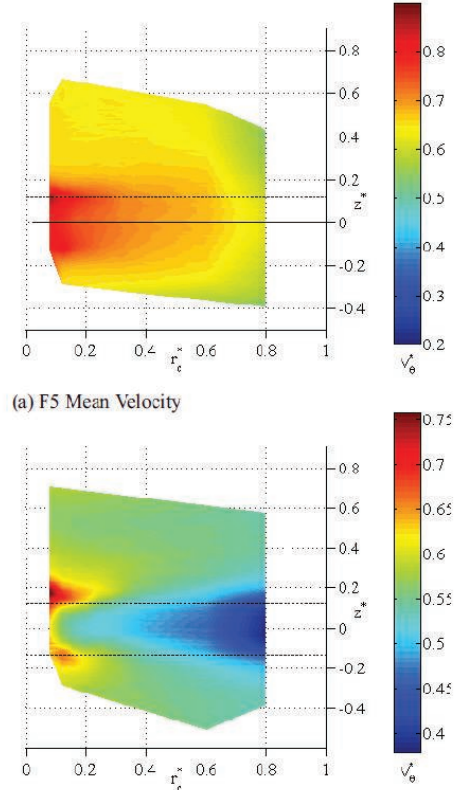

(c) R5 Mean Velocity
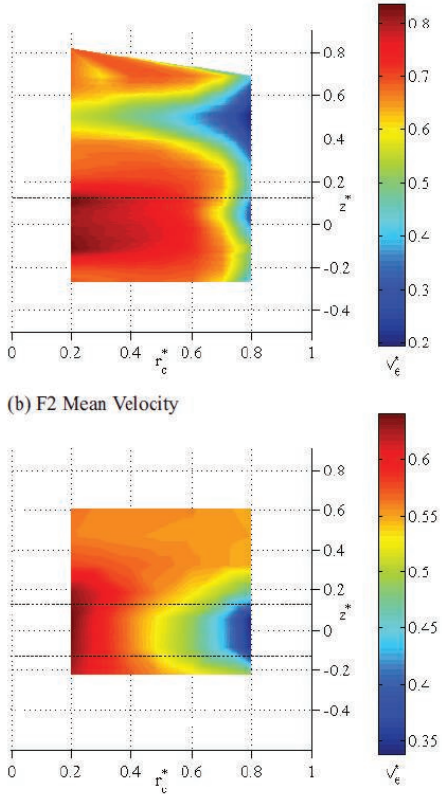

(d) R2 Mean Velocity

Fig. 5: Normalized mean velocity distributions $V_{\theta}$ with color codes in the tip gap region for the four different cases: (a) F5; (b) F2; (c) R5; (d) R2. The positions of the two disks are shown as broken lines in the plots. The disk edge corresponds to $r_{c}=0$ and the shroud wall is at $r_{c}=1$.

(a)

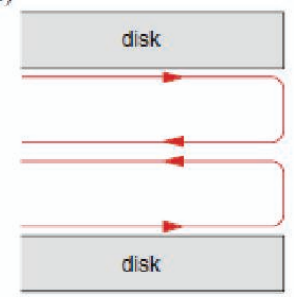

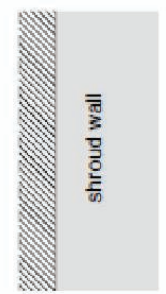

(b)

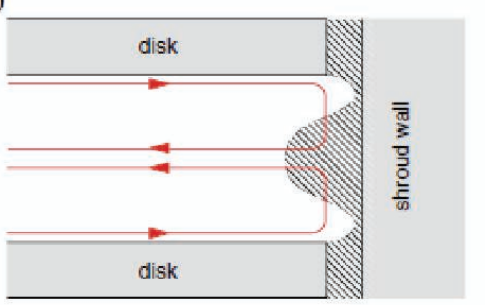

Fig. 6: Sketch of the secondary flow pattern in the boundary layers: (a) case F5, (b) case F2. The shaded area indicates the boundary layer on the shroud.

\section{Concluding Summary}

The flow behaviors in the tip clearance region of rotating disks and the shroud wall was investigated for the first time using laser Doppler techniques: a conventional LDV and the velocity profile sensor. The velocity measurements of the circumferential component were carried out for the four different experimental configurations with the combination of two different gap widths and shroud shapes with and without ribs. The complex flow velocity structures were successfully captured with the advantage of the high spatial resolution provided by the velocity profile sensor. The resulting velocity statistics revealed the existence of a highly distorted three-dimensional boundary layer in the clearance region due to the strong shear forces working in the two orthogonal directions merging at the sharp corner of the disk edge. The velocity behaves significantly different around the inter disk region near the shroud surface for the two different gap widths with a flat shroud shape. When the tip clearance becomes smaller, the boundary layers developing over the disk surfaces interact with another boundary layer developed over the shroud wall. It turned out that the reduction of the turbulence fluctuation using the ribbed shroud is limited to specific region and the ribbed shroud does not necessarily reduce the fluctuation in total. The next step of the investigation is preapred in oder to investigate the unsteady flow behaviers in a HDD including the read-and- 
write arm and the shroud openings existing in a real HDD. The flow with such obstructions may exhibit strong interaction with the boundary layer developed over the disk and shroud surface found in the present study.

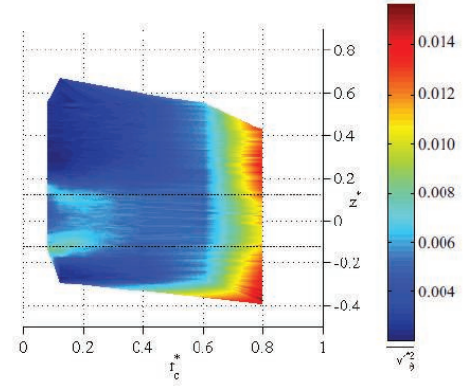

(a) F5 Velocity Variance

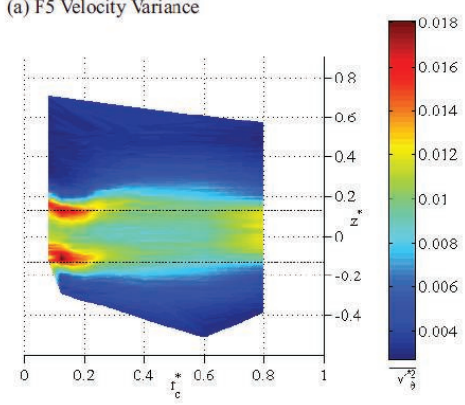

(c) R5 Velocity Variance
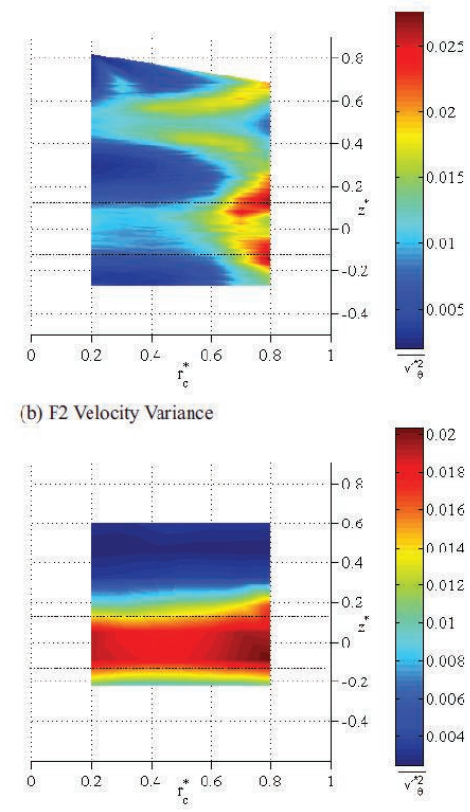

(d) R2 Velocity Variance

Fig. 7: Normalized velocity variance distributions ${\overline{v_{\theta}}}^{*}$ with color codes in the tip gap region for the four different cases: (a) F5; (b) F2; (c) R5; (d)R2. The positions of the two disks are shown as broken lines in the plots. The disk edge corresponds to $r_{c}=0$ and the shroud wall is at $r_{c}=1$.

\section{Acknowledgements}

This research was financially supported by the Storage Research Consortium (SRC), the Deutsche Akademische Austauschdienst (DAAD) and the Deutsche Forschungsgemeinschaft (CZ55/20-1,2). The support are greatly appriciated. Mr. Yusuke Yaguchi is acknowledged for his carrying out the experiment.

\section{References}

[1] S.D. Abrahamson, J.K. Eaton, D.J. Koga (1989) The flow between shrouded corotating disks. Phys Fluids A, Vol. 1, pp. 241-251.

[2] S. Imai, M. Tokuyama, Y. Yamaguchi (1999) Reduction of disk flutter by decreasing disk-to-shroud spacing. IEEE Trans Magnetics, Vol. 35, pp. 2301-2303.

[3] Y. Yamamoto, S. Obi, S. Masuda (2004) Effect of disk-shroud spacing on flow induced vibration in corotating disk system. Proc ASME Information Storage and Processing Systems Conference, June, 2004, Santa-Clara, USA.

[4] J. Czarske (2001) Laser Doppler velocity profile sensor using a chromatic coding. Meas Sci Technol, Vol. 12, pp. 52-57.

[5] J. Czarske, L. Büttner, T. Razik, H. Müller (2002) Boundary layer velocity measurements by a laser Doppler profile sensor with micrometre spatial resolution. Meas Sci Technol, Vol. 13, pp. 1979-1989.

[6] J. König, A. Voigt, L. Büttner, J. Czarske (2010) Precise micro flow rate measurements by a laser Doppler velocity profile sensor with time division multiplexing, Meas Sci Technol, Vol. 21, 074005 (9 pp).

[7] L. Büttner, C. Bayer, A. Voigt, J. Czarske, H. Müller, N. Pape, V. Strunck (2008) Precise flow rate measurements of natural gas under high pressure with a laser Doppler velocity profile sensor. Exp Fluids, Vol. 45, pp. 1103-1115.

[8] K. Shirai, C. Bayer, A. Voigt, T. Pfister, L. Büttner, J. Czarske J (2008) Near-wall measurements of turbulence statistics in a fully developed channel flow with a novel laser Doppler velocity profile sensor. Euro J Mech B/ Fluids, Vol. 27, pp. 567-578.

[9] C. Bayer, K. Shirai, L. Büttner, J. Czarske (2008) Measurement of acceleration and multiple velocity components using a laser Doppler velocity profile sensor. Meas Sci Technol, Vol. 19, 055401 (11 pp).

[10] K. Shirai, Y. Yaguchi, L. Büttner, J. Czarske, S. Obi (2010) Highly spatially resolving laser Doppler velocity measurements of the tip clearance flow inside a hard disk drive model. Exp Fluids (in print).

[11] T. Pfister, L. Büttner, K. Shirai, J. Czarske (2005) Monochromatic heterodyne fiber-optic profile sensor for spatially resolved velocity measurements using frequency division multiplexing. Appl Optics, Vol. 44, pp. 25012510. 